Iberian Journal of the History of Economic Thought

ISSN-e 2386-5768

\title{
Breve opúsculo de las obras de autores españoles que, entre 1861 y 1905 , estudiaron cuestiones de naturaleza monetaria
}

José Ramírez Rabanal ${ }^{1}$

La lectura del Crédito papel (1802) $)^{2}$, una obra en la que, hace más de dos siglos, Henry Thornton plantea cuestiones que aún hoy son de actualidad, despertó mi interés por conocer cómo se pensaba en España en el siglo XIX sobre cuestiones de naturaleza monetaria. Dicho interés me llevó a buscar e indagar sobre el pensamiento monetario decimonónico español y las ideas y autores españoles que trataran sobre tales cuestiones. Así, descubrí obras de autores españoles, algunos de ellos poco conocidos o nombrados, que entre 1861 y 1905 escribieron sobre el proceso que condujo a la proscripción de la moneda de plata como instrumento de pago internacional, relacionado con la crisis del bimetalismo y la preferencia por el patrón único oro. Al margen de este tema, esos autores también trataron en sus obras otras cuestiones de carácter monetario que se plantearon en el último tercio del siglo XIX.
Así pues, los objetivos de este breve ensayo son recopilar y ordenar, de forma muy resumida, las opiniones, planteamientos y propuestas recogidos en algunas de las obras de estos autores del XIX e intentar demostrar, en contra de la tesis tradicional foránea que descansa en la práctica ausencia del pensamiento económico español en los debates monetarios de la época, que sí hubo autores españoles que aportaron cuestiones interesantes al pensamiento económico del siglo XIX.

Los planteamientos, opiniones y propuestas de estos autores aquí resumidos, se encuentran desarrollados y comentados en mi tesis doctoral "Causas y efectos de la crisis monetaria internacional de mediados del XIX (1861-1895), según los autores españoles de la época".

Cabe señalar, por último, que la paginación que aquí se incluye y acompaña algunas de las citas corresponde, en cada caso, a la obra citada y brevemente comentada de forma debida. 


\begin{tabular}{|l|c|}
\hline \multicolumn{2}{|c|}{ ÍNDICE DE AUTORES } \\
\hline \multicolumn{2}{|c|}{ Página } \\
\hline AUTOR ANÓNIMO & 86 \\
\hline BARTHE Y BARTHE, Andrés & 86 \\
\hline BARROETA ALDAMAR, Joaquín Francisco de & 87 \\
\hline FERNÁNDEZ VILLAVERDE, Raimundo & 87 \\
\hline FIGUEROLA Y BALLESTER, Laureano & 87 \\
\hline GENER, José & 88 \\
\hline GIL Y PABLOS, Francisco & 88 \\
\hline GIRONA Y AGRAFEL, Manuel & 89 \\
\hline GÜELL Y FERRER, Juan & 89 \\
\hline HERIZ, Enrique & 89 \\
\hline JIMÉNEZ Y RODRÍGUEZ, José María & 89 \\
\hline NAVARRO REVERTER, Juan & 90 \\
\hline ORTÍ Y BRULL, Vicente & 90 \\
\hline PARET Y GUASP, Lorenzo Victor & 91 \\
\hline RUÍZ GÓMEZ, Servando & 91 \\
\hline SÁNCHEZ DE TOCA, Joaquín & 91 \\
\hline SANROMÁ Y CREUS, Joaquín María & 92 \\
\hline SANZ Y ESCARTÍN, Eduardo & 92 \\
\hline TUTAU Y VERGES Juan & 92 \\
\hline VÁZQUEZ QUEIPO DE LLANO, Vicente & 93 \\
\hline VIVER, Eudaldo & 93 \\
\hline
\end{tabular}

\section{AUTOR ANÓNIMO (1877)}

La cuestión monetaria en España, Establecimiento tipográfico de Baseda y Giró, Barcelona, 1877. No se ha logrado identificar al autor de esta obra, catalogada en la Biblioteca Nacional de España con la signatura VC/606/18.

En dicha obra se plantean los problemas derivados de la inestabilidad del valor relativo entre el oro y la plata, llegando a la conclusión de que, ante la imposibilidad de poder fijar por ley dicho valor relativo, es necesaria la adopción de un solo patrón monetario sobre la base del oro. También trata los efectos que sobre el precio del oro y la plata tienen los excedentes de producción de ambos metales, procedentes de las nuevas minas. La opinión del autor sobre dicho exceso de producción es que no se producirán efectos relevantes, ya que los excedentes serán absorbidos por las necesidades derivadas del aumento de la población, por el desarrollo de la actividad económica y por la necesidad de crear las reservas metálicas necesarias para garantizar las emisiones de billetes. En cuanto a la cuestión monetaria en España, el libro examina los objetivos de las reformas y de las disposiciones monetarias adoptadas entre 1848 y 1877 , prestando especial atención a la reforma de 1868 .
BARTHE Y BARTHE, Andrés (1860-?). Premio de la Real Academia de Ciencias Morales y Políticas por su Memoria de 1897 y premio de la Asociación de Profesores Mercantiles por su Memoria de 1903.

Estudio crítico de la crisis monetaria en España, Establecimiento Tipográfico de Jaime Ratés, Madrid, 1905. En esta obra se considera, en primer lugar, la salida de la plata de España en 1859 como consecuencia de su menor valor respecto al oro, en comparación con el existente en otros países vecinos. Y, en segundo lugar, las causas de la salida del oro de España en 1874 y de 1879 a 1891 y por qué no se hizo nada para evitar la escasez de oro que estas salidas provocaban. Además, en esta obra se facilitan interesantes estadísticas sobre la evolución de la circulación monetaria en España entre 1867 y 1895.

El problema monetario, Imprenta de Fortanet, Madrid, 1908. Barthe y Barthe reconoce aquí que la plata circula en España en cantidad muy superior a la que exige el mercado interior, por lo que considera necesario poner la circulación monetaria española en equilibrio con la de otros países donde circula el oro. Por otra parte, da a entender que el exceso de acuñaciones de plata que se produce en España 
está alentado por los beneficios que el Tesoro obtiene con dichas acuñaciones.

BARROETA ALDAMAR, Joaquín Francisco de (1796-1865). Senador (1844), nombrado vitalicio en 1860. Consejero del Banco Español de San Fernando.

Informe sobre moneda; Memoria sobre el arreglo del sistema monetario en España, Imprenta Nacional, Madrid, 1861. Se recogen propuestas y las conclusiones derivadas de un Informe que, con el propósito de aclarar las circunstancias que provocaban la salida de España de la moneda de plata gruesa, le fue encargado a Barroeta Aldamar por Salaverría en 1858. En este informe se atribuye la salida de plata de España a la exportación de los duros españoles y a la diferencia existente entre nuestro valor relativo oro/ plata y el vigente en otros países. Como solución a dicho problema, Aldamar propone la adopción del patrón único oro. Quizás, lo más interesante de esta obra sea el análisis que hace el autor del efecto que, sobre las transacciones comerciales y los precios, provoca el aumento de numerario. Un análisis que se ajusta a la teoría cuantitativa del dinero ya que admite no sólo la contribución de la abundancia de oro al desarrollo de la actividad económica, sino que dicha abundancia de numerario provoca forzosamente el aumento de los precios. También es interesante el amplio análisis realizado sobre el efecto que la abundancia de oro y plata tuvo sobre el nivel general de los precios entre 1850 y 1870.

\section{FERNÁNDEZ VILLAVERDE, Raimundo} (1848-1905). Profesor supernumerario de la Universidad Central de Madrid (1859). Gobernador civil de Madrid (1884). Ministro de Gracia y Justicia (1890). Ministro de Gobernación (1892). Ministro de Hacienda (18991900). Presidente del Consejo de Ministros (1903). Presidente del Congreso de los Diputados.

La cuestión monetaria, en Memorias de la Real Academia de Ciencias Morales y Políticas, Tomo VII. (pp. 23 a165), Imprenta y Litografía de los Huérfanos, Madrid, 1893. En este trabajo, Fernández Villaverde se basa en las conclusiones del Informe de la Comisión del Oro y de la Plata, presentado en 1889 para analizar cuestiones muy debatidas a mediados del XIX, tales como las causas de la caída generalizada de los precios, considerada como origen de la crisis económica del último tercio del siglo XIX y que según Fernández Villa- verde está provocada por la revalorización del oro al quedar, tras la proscripción internacional de la plata, como el único instrumento disponible para efectuar los pagos al extranjero; la discusión sobre la adopción de un sistema monetario basado en un patrón único o, alternativamente, en un doble patrón; las causas de la depreciación de la plata y sus graves consecuencias, sobre todo en aquellos países en los que, como España, la plata era la base de su circulación monetaria; las propuestas presentadas en las diversas Conferencias, celebradas en 1878,1881 y 1889 con el objetivo de conseguir la rehabilitación internacional de la moneda de plata, con el que el autor se manifiesta en desacuerdo, pues opina que el desarrollo del billete y del crédito pueden cubrir el vacio dejado por la plata; y finalmente, las consecuencias de la reforma de 1868 efectuada en España que, junto con los desaciertos derivados de las disposiciones de 1871, supusieron, a juicio de Fernández Villaverde, un error por el que el oro emigró o fue atesorado y a cambio nos vimos inundados de plata. Además, cabe destacar las interesantes series estadísticas presentadas en las páginas 141 a 167 de esta obra.

FIGUEROLA Y BALLESTER, Laureano (1816-1903). Catedrático de Derecho Administrativo y Economía Política en la Universidad de Barcelona (1845). Catedrático de Economía Política, Derecho Político y Legislación Mercantil de la Universidad Central de Barcelona (1851) y posteriormente en la Central de Madrid (1853). Académico de la Real Academia de Ciencias Morales y Políticas. Fundador de la Sociedad Libre de Economía Política (1857). Diputado a Cortes (1854-1856). Senador (1871-1873), Presidente de la Cámara Alta (1872). Ministro de Hacienda (1868).

Laureano Figuerola impulsó y promulgó la Reforma Monetaria de 1868. En la exposición de motivos de dicha Reforma se señala que: "El triunfo de la revolución[...] hace indispensable[...] la reacuñación de la moneda[...] Pero al reacuñar la moneda[...] parece ocasión oportuna de realizar la reforma del sistema monetario, ajustando éste a las bases adoptadas en el convenio internacional de 23 de Diciembre de 1865 por Francia, Bélgica, Italia y Suiza[...]" Aclarando al mismo tiempo "[...] que España no entra a formar parte de la Unión Monetaria Latina hasta que se halle constituido definitivamente el país y reanudadas las relaciones diplomáticas con los demás pueblos". 
["Exposición de motivos" del Decreto del 19 de octubre de 1868, Gaceta de Madrid, n 294, (20 de octubre de 1868), p. 1].

Discusión sobre el estado anormal de los cambios, Tomo VII de las Memorias de la Real Academia de Ciencias Morales y Politicas, Imprenta y Litografía de los Huérfanos, Madrid, 1893, pp. 517 a 575. En tal Discusión intervienen, además de Figuerola, los señores Gumersindo Azcárate, Melchor Salvá, Luis María de la Torre y de la Hoz (Conde de Torreanaz), Fernando Cos-Gayón, Joaquín Sánchez de Toca, Manuel Colmeiro y Fernández Villaverde, y en ella se tratan básicamente dos problemas monetarios: las causas de la elevación del tipo de cambio en España y de su escasez de oro. Asuntos en los que los autores expresan sus opiniones y aportan sus particulares remedios. ${ }^{3}$

GENER, José. Director General de Consumos, Casas de Monedas y Minas (1862).

Informe sobre la cuestión monetaria elevado al Sr. Ministro de Hacienda. Imprenta Na-

Aunque todos ellos sobradamente conocidos (algunos destacados economistas del pensamiento económico español) en los debates sobre el patrón monetario, el sistema monetario internacional y otras cuestiones monetarias, ni Azcárate, ni Salvá, ni el conde de Torreanaz, ni CosGayón, ni Colmeiro aparecen citados en este trabajo por no aportar obras propias o exclusivas sobre tales cuestiones monetarias. No obstante, en mi investigación doctoral antes referida se recogen sus opiniones y propuestas sobre tales asuntos y materias. Así, sobre el problema de la causa de la elevación del tipo de cambio, Figuerola, Salvá, Sánchez de Toca y Fernández Villaverde, parecen estar de acuerdo en que el factor esencial del quebranto del cambio en España es la prima que hay que pagar por el oro escaso. El Conde de Torreanaz considera, sin embargo, que las variaciones del cambio obedecen a nuestro exceso de demanda de valores y al déficit comercial con el exterior. Cos-Gayón opina que la causa de la elevación del cambio no se debe al déficit comercial con el exterior ni al déficit presupuestario, sino a la falta de confianza en nuestra Deuda que impide emitirla para cancelar nuestros saldos con el exterior, lo que nos obliga a recurrir a la compra de oro. Para Navarro Reverter - véase más adelante- es el precio de las letras pagaderas en oro lo que determina la subida del cambio. Sanz y Escartín, también citado aquí, mantiene que el quebranto del cambio es consecuencia de la depreciación de la moneda interior pues, según él, todos los quebrantos en los cambios obedecen a deficiencias de valor intrínseco en la circulación y nunca a desequilibrio de la balanza económica. Figuerola va un poco más allá y, sobre la causa de la escasez de oro, argumenta que el actual malestar nace no solo de la mayor cantidad del metal de plata, sino del exceso de su acuñación y que, en todas partes, el billete sustituye a la moneda y su abuso ha ahuyentado al oro, encareciéndolo. Azcárate también atribuye la escasez de oro al exceso de circulación fiduciaria, aunque añade que también contribuyó a ello el pago de la deuda exterior, refiriéndose probablemente al pago de los títulos que nos fueron devueltos por los acreedores en 1891 cional, Madrid, 1862. Esta obra, tiene por objeto determinar las posibles perturbaciones que la afluencia de oro y la desaparición de la plata podrían provocar en España. Sobre la afluencia de oro, Gener opina que la abundancia de oro no podrá ser absorbida por lo que es inevitable que su excedente conduzca a la caída de los precios (del nivel general de precios). En cuanto a la desaparición de la plata provocada por la inestabilidad de su valor relativo, después de analizar varias alternativas y descartada la posibilidad de mantener estable dicho valor relativo entre el oro y la plata, Gener llega a la conclusión de que ninguna de las medidas planteadas puede resolver dicho problema. Por tanto, ante esta situación, Gener propone una reforma del sistema monetario consistente en aumentar el fino de las monedas de oro, mediante su necesaria refundición general. Sin embargo, al reconocer más adelante que la refundición general de las monedas de oro tampoco resolvería el problema, Gener llega a la conclusión de que la única solución posible sería adoptar en España un único patrón monetario. Empero, al no haber acuerdo sobre cuál debía ser el metal elegido, dado que mientras Vázquez Queipo propone la plata, Aldamar se inclina por oro, Gener termina proponiendo una reforma del sistema monetario que llevaría a la Reforma de 1864.

GIL Y PABLOS, Francisco (1877-1918). Doctor en Leyes. Hombre de negocios relevante y colaborador del diario El Economista.

Estudios sobre la moneda y los cambios. Imprenta de la Revista de Legislación, Madrid, 1906. El libro trata, en primer lugar, el efecto que tuvo el notable incremento de la producción de oro y plata a mediados del XIX sobre sus respectivos precios, así como sobre su tasa de cambio, manifestando el autor que tal incremento no afectó al precio de los metales, ya que estos estuvieron sostenidos por el aumento de la actividad comercial que generaba la abundancia de los mismos. Además, se estudia el efecto que sobre la actividad económica y los precios tuvo el aumento de la producción de metales entre 1848 y 1870 . Sobre este particular Gil y Pablos considera que la transformación económica que se produce a mediados del XIX "fue alentada, esto es indudable, por el aumento de las especies metálicas; pero no menos cierto [es] que contribuyó a ese resultado el inevitable despertar del letargo en que yacía el mundo económico en la primera 
mitad del XIX." (p. 96); y establece que fue esta transformación económica la que llevó al alza del nivel general de precios. Un alza de precios que, basándose en que no han subido con la misma intensidad en todas las mercancías, el autor considera que no puede atribuirse a un solo factor, sino a factores diferentes, como pueden ser la subida de los salarios y la abundancia de numerario, si bien Gil y Pablos insiste en que no fue esta la causa más influyente. La obra dedica también dos capítulos al proceso de depreciación de la plata, analizando sus causas y consecuencias así como los esfuerzos de EE.UU. y de la Liga bimetalista inglesa para rehabilitar el uso monetario de la plata en Europa. Contiene, finalmente, la obra otros capítulos interesantes dedicados a la relación entre la contracción monetaria y la baja de precios a finales del XIX; el proceso hacia la adopción del sistema del patrón oro y la cuestión monetaria en España, donde se examinan el Decreto de 1868 y las causas del estado del tipo de cambio en España así como los remedios para mejorarlo.

GIRONA Y AGRAFEL, Manuel (18171905). Banquero y político. Director del Banco de Barcelona. Senador. Fundador y Presidente de la Cámara de Comercio Industria y Navegación de Cataluña entre 1886 y 1901.

Ensayos para mejorar el crédito y arreglar la situación de España, Est. Tipográfico de N. Ramírez y Rialp, Barcelona, 1865. Basándose en la afirmación de que "[...] el crédito es la poderosa palanca de la época[...] su prudente uso es beneficioso[...] su abuso es devastador[...]" (p. 20), el autor intenta demostrar que la desastrosa situación monetaria de España está determinada por haber abusado de la emisión de billetes, por lo que propone en esta obra soluciones para resolver dicho problema.

GÜELL Y FERRER, Juan (1800-1872). Industrial y economista. Hombre de negocios creó varias empresas y fue uno de los fundadores del Banco de Barcelona.

Examen de la crisis actual con ocasión del opúsculo publicado por[...] D. Vicente Vázquez Queipo, Imprenta Narciso Ramírez y C., Barcelona. 1866. En el opúsculo que cita esta obra, Vázquez Queipo manifiesta su opinión de que la causa de la crisis monetaria en España de 1866 estaba determinada por la escasez de numerario metálico y que, tal escasez, estaba provocada por el incremento conside- rable de su demanda como consecuencia del descredito que sufría el papel fiduciario debido a su ilimitada emisión. En su Examen, Güell y Ferrer coincide con Vázquez Queipo en que la crisis monetaria se produce por una escasez de numerario metálico, pero discrepa en cuanto a la causa de la escasez de metálico, ya que considera probado que dicha escasez es consecuencia de los pagos del déficit de la balanza comercial.

HERIZ, Enrique. Escritor científico.

Memoria sobre la unidad monetaria, Tipografía de Narciso Rodríguez, Madrid, 1873. Aquí se plantean las dificultades que supone la implantación del doble patrón aunque, al mismo tiempo, se indican tres maneras bajo las cuales puede llevarse a cabo el doble patrón, que son: que el Estado fije una relación entre el oro y la plata y refunda una de las dos monedas; evitar las refundiciones y que el estado varíe la relación del valor entre el oro y la plata; y acuñar monedas de oro y plata del mismo peso y ley, dejando que su relación oscile según la ley de la oferta y la demanda. Los datos que Enrique Heriz facilita (pp. 35 a 37) sobre la evolución de las producciones de oro y plata desde la antigüedad son realmente interesantes.

\section{JIMÉNEZ Y RODRÍGUEZ, José María} (1859-?). Economista. A partir de 1896 dirigió varias sucursales del Banco de España.

Estudio crítico de la cuestión monetaria, Tipografía de Jaime Ratés, Madrid, (1905). La sección primera, parte primera, de la obra determina las causas de la crisis monetaria internacional provocada por la depreciación de la plata, distinguiendo entre causas eficientes, que son aquellas que han producido directamente la crisis, y causas formales, que son las que determinan la importancia o intensidad de la crisis en cada país. Respecto de las causas eficientes, el autor considera que la depreciación de la plata ha sido la causa general de la crisis iniciada a mediados del XIX y sostiene que dicha depreciación se inicia con la adopción del patrón oro por parte de Alemania, aunque serán las disposiciones de la Unión Monetaria Latina de 1873 y 1878 lo que rompa el equilibrio de valor entre el oro y la plata.

En cuanto a las causas formales considera algo trascendental las deudas internacionales de un país en relación con los medios de los que dispone para amortizarlas. Así, en aquellos 
países que no dispongan de oro ni de mercancías para cancelar sus deudas, resultará que la demanda de letras sobre el extranjero excederá a su oferta, lo que supondrá la elevación (depreciación) de su tipo de cambio, que favorecerá sus exportaciones, estimulará su producción y limitará las importaciones. Pero, al mismo tiempo, la elevación del tipo de cambio conducirá a un alza de los precios interiores que obligará a un aumento de la moneda en circulación. Concluye el autor que el aumento de las exportaciones, de la producción y de la moneda en circulación "[...] han producido el efecto de despertar la actividad de las naciones deudoras, y ponerlas en condiciones de dejar de serlo" (p. 44).

La segunda sección analiza los efectos de la crisis que, evidentemente, el autor considera se derivan de la falta de oro ante la cual los países deudores, especialmente los monometalistas de plata y los bimetalistas, se vieron obligados a comprar oro con una prima aproximadamente igual al quebranto que la moneda de plata sufría en el extranjero, provocando oscilaciones de los cambios que, a su vez, favorecieron un aumento de la producción y una disminución del consumo en los países con circulación basada en la plata, mientras que en los países con circulación basada en el oro se produjo una disminución de las exportaciones y un aumento de sus importaciones. Y la tercera sección trata de la solución de la crisis y aquí el autor considera que, para evitar su agravamiento, los Gobiernos deben actuar sobre la cantidad de moneda circulante, las deudas exteriores, las alteraciones de los cambios y el fomento de la producción.

En la parte segunda del libro, Jiménez y Rodríguez investiga las causas y los efectos de la crisis monetaria en España que, según él, estuvo determinada por la depreciación de la plata y las deudas exteriores como causa eficiente, pero que se agravó por dos causas formales tales como los aranceles librecambistas de 1869 y 1882, que facilitaron las importaciones y detuvieron el progreso de la producción, y la insuficiente producción española en relación con sus necesidades de consumo, circunstancias que obligaron a España a utilizar sus stocks de oro y provocaron la escasez de dicho metal. La escasez de oro supuso, a su vez, el pago de una elevada prima por el oro, que implicaba la elevación (depreciación) del tipo de cambio que, afirma el autor, habría conducido a un aumento generalizado de los precios interiores; a favorecer las exportaciones, estimulando la producción; a limitar las importaciones; a la reducción del consumo; y a la necesidad de aumentar la circulación fiduciaria, así como las acuñaciones de plata para compensar el vacío dejado en la circulación por el oro.

NAVARRO REVERTER, Juan (1844-1924). Ingeniero. En 1877 fundó la Caja de Ahorros y Monte de Piedad de Valencia. Diputado y Senador. Ministro de Hacienda entre 1895 y 1897. Desde 1916 Presidente de la Compañía Arrendataria del Monopolio de Tabacos.

En su libro El cambio internacional, Establecimiento Tipográfico Hijos de J.A. García, Madrid, 1903, se intenta dar solución al problema de los tipos de cambio con el extranjero. Un problema que viene determinado por la desmonetización internacional de la plata, que lleva a la necesidad de realizar los pagos al exterior en oro. Por lo que si no se tiene oro, como era el caso de España, hay que pagar en plata, añadiendo una prima equivalente a la diferencia entre su precio y el del oro. Relata Navarro Reverter que para resolver dicho problema se han propuesto varias soluciones, entre las que destaca reducir los billetes en circulación, desmonetizar la plata o el Plan propuesto por el Gobierno, consistente en el pago en oro de los derechos de aduana. Soluciones todas que el autor descarta porque, dice, no existe relación entre el cambio y la circulación de billetes y porque no está de acuerdo con la desmonetización de la plata, dado que siempre ha mantenido que las acuñaciones de plata salvaron a España de tener que recurrir al billete forzoso. En cuanto al Plan del Gobierno, critica tanto lo que se refiere a las medidas para reducir la circulación de los billetes, como lo relativo al pago en oro de los derechos de aduana. Por tanto, y como solución al problema del cambio, considera que lo mejor sería adoptar el patrón oro si bien reconoce que no es fácil ejecutar tal medida cuando no se dispone de oro en la circulación.

ORTÍ Y BRULL, Vicente (?-1897). Escritor. Inspector de sucursales en el Banco de España.

La cuestión monetaria, Librería de Fernando Fe, Madrid. 1893. Ortí y Brull critica en este libro las teorías monometalistas y defiende el bimetalismo al considerar las fatales consecuencias provocadas por las limitaciones, impuestas a partir de 1873, para el uso monetario de la plata. Por ello, defiende la rehabilitación del uso monetario de la plata en toda Europa, 
lo que le lleva a examinar ampliamente el desarrollo de las conferencias celebradas con este fin, a instancias de EE.UU. y Francia, en 1878, 1881,1889 y 1892 . Concretamente, sobre las causas de la depreciación de la plata considera que fueron determinantes las limitaciones a las acuñaciones de plata impuestas por la Unión Monetaria Latina en 1873 y 1878, poniendo en duda - además - que los nuevos instrumentos de crédito hubieran cubierto el vacío dejado en la circulación por la moneda metálica. Por esta razón considera que las limitaciones impuestas a las acuñaciones de plata, unidas a la escasez de moneda de oro, fue lo que provocó la contracción monetaria causante de las crisis económicas del último tercio del XIX (18731893). Sobre la razón de la salida de oro en España a finales del XIX, atribuye la misma al pago de los títulos de la Deuda exterior que nos fueron devueltos por sus tenedores como resultado de la desconfianza provocada por la campaña de descredito emprendida por la banca francesa sobre dichos títulos.

PARET Y GUASP, Lorenzo-Víctor (18791954). Profesor Mercantil (1897). Presidente del Colegio Central de Profesores Mercantiles.

Encarecimiento de la vida en los principales países de Europa y singularmente en Espa$\tilde{n} a$, Establecimiento Tipográfico de Jaime Ratés, Madrid, 1914. El libro trata ampliamente las causas del encarecimiento de la vida entre 1881 y 1909. Según Paret y Guasp, sobre el asunto se han realizado trabajos que atribuyen el encarecimiento al aumento de la producción del oro, al proteccionismo, a la influencia de las reformas sociales y al crecimiento de las cargas públicas. Pero él no está de acuerdo con todas esas teorías, pues considera que son varias las causas que actúan sobre dicho encarecimiento. Su conclusión es que la producción del oro tiene poca influencia sobre el encarecimiento y que tienen mayor incidencia el urbanismo y los gastos públicos pero, sobre todo, el proteccionismo y el socialismo, que son factores que ejercen influencia sobre los precios elevándolos. Resultan dignas de mención las series estadísticas que recoge la obra.

RUÍZ GÓMEZ, Servando (1821-1888). Miembro de la Junta de Fomento en la Habana (1848). Diputado y Senador. Ministro de Hacienda (1872).

La cuestión monetaria, Sucesores de Ribadeneyra, Madrid, 1892. El autor critica en este libro el abuso que, por codicia e imprevisión, se hizo en España de la acuñación de plata, mientras que los países pudientes la disminuyeron. A propósito de la Conferencia de 1865, en la que Francia, Bélgica, Suiza e Italia acordaron la uniformidad de sus sistemas monetarios (Unión Monetaria Latina), opina que la ausencia de España en dicha Conferencia fue para no verse sometida a las obligaciones que en ella se acordaron relativas al peso y ley de las acuñaciones de monedas de oro y plata, así como los límites establecidos sobre la cuantía de monedas divisionarias de plata en cada pago o la fijación de la cuantía de las emisiones de monedas divisionarias de plata. Reconoce la gravedad de la situación monetaria en España provocada por la depreciación de la plata, encontrándonos aislados y atascados en la acuñación de plata mientras sigue saliendo el oro del país. Se pregunta por qué baja tanto el precio de la plata frente al del oro. Su respuesta es que las acuñaciones de oro han crecido 17 veces, mientras que las de plata han disminuido 2,7 veces, lo que le lleva a la conclusión de que la baja del precio de la plata se debe a la caída de su demanda para acuñaciones. Sobre la situación de los cambios en España critica la reforma de 1868 por no haber limitado la acuñación de plata y los pagos al exterior en esta moneda, ya que ello supone pagar un precio más alto por ser un metal menos apreciado.

SÁNCHEZ DE TOCA Y CALVO, Joaquín (1852-1942). Ministro de Agricultura, Industria y Comercio (1900). Ministro de Marina (1902). Ministro de Gracia y Justicia (19031904). Presidente del Consejo de Estado (1904). Presidente del Consejo de Ministros (1919). Presidente del Senado (1919).

El oro, la plata y los cambios, Tipografía de los Hijos de M. G. Hernández, Madrid, 1894. La obra analiza el proceso de desmonetización internacional de la plata, sus efectos en Europa y, especialmente, sus repercusiones sobre los cambios y la economía española. Respecto de la situación monetaria en España, Sánchez de Toca está en contra de quienes proponen prescindir de las acuñaciones de plata y adoptar el patrón único oro, porque considera que la circulación de la moneda de plata constituye un elemento importante de la defensa económica de nuestro país por varias razones. Primero, el oro no ha salido porque haya sido expulsado por la plata sino porque alcanzó su punto de salida, siguiendo análisis 
clásicos al uso. Además, la circulación monetaria de plata ha sido el mejor freno a las bruscas oscilaciones de los tipos de cambio con el extranjero. Y por último, las acuñaciones de plata, tal y como se han llevado a cabo en España durante los últimos años, en nada han contribuido al alza de los cambios. Por todo ello, concluye que si careciéramos de existencias de plata y el oro despareciera de la circulación nos quedaríamos reducidos al "papel" como único instrumento de pago, que además se impondría con curso forzoso al carecer de la indispensable cobertura metálica. Atribuye Sánchez de Toca el déficit endémico exterior de España no sólo al hecho de que las importaciones superen a las exportaciones sino, sobre todo, a "la importación invisible de capitales" (p. 119). Según él, esto obligó a España a cubrir sus déficits con la emisión de deuda exterior y fue la necesidad de atender el pago de dicha deuda lo que provocó la subida de nuestro cambio con el extranjero. No obstante, reconoce el efecto que la elevación del tipo de cambio tuvo sobre la economía española favoreciendo las exportaciones, la producción y el consumo, y limitando las importaciones. Finalmente, trata la importancia de nivelar el presupuesto nacional mediante el crédito público y las instituciones bancarias para evitar tener que recurrir al crédito internacional, y tener que pagar altos tipos de interés, aunque reconoce que todavía en España la gente desconfía del crédito y prefiere ocultar los caudales.

SANROMÁ Y CREUS, Joaquín María (1828-1895). Catedrático de Economía Política, Derecho Político y Administración de la Universidad de Santiago de Compostela. Catedrático de Historia General del Comercio y Elementos del Derecho Internacional Mercantil de la Escuela Profesional de Comercio de Madrid. Diputado en Cortes (1871-1874). Consejero de Estado y de Instrucción Pública (1891).

La Cuestión Monetaria en España, Imprenta de T. Fortanet, Madrid, 1872. Este libro hace referencia a la Conferencia de París de 1865 y reconoce su importancia al intentar la unificación monetaria internacional. En el mismo se consideran también los objetivos e inconvenientes de la reforma de $1864 \mathrm{y}$, sobre todo, los problemas que planteó la no aplicación completa de la reforma de 1868 , tales como no haber recogido y reacuñado el numerario antiguo de ambos metales, de mayor ley o peso fino, con el fin de igualarlos al peso y fino de las nuevas acuñaciones.

La Conferencia Monetaria de 1881, Tipografía de Manuel G. Hernández, Madrid. 1881. Sanromá trata en esta obra, de manera muy amplia, el desarrollo y fracaso de las Conferencias de Paris de 1878 y 1881, destinadas a intentar la rehabilitación de la plata en Europa. En concreto, sobre la Conferencia de 1881, son interesantes los planteamientos que hace el autor en torno a las causas de la depreciación de la plata que atribuye, principalmente, al aumento de su producción. Pero, tal vez, una de las aportaciones más interesante que hace Sanromá es la de reconocer la importante contribución del papel moneda, así como de los nuevos instrumentos de crédito para suplir en la circulación monetaria a la moneda de plata.

SANZ Y ESCARTÍN, Eduardo (1855-1939), Gobernador y Senador por Granada en 1903. Gobernador del Banco de España (19191921). Ministro de Trabajo (1921). Presidente del Instituto de Estudios Sociales (1921-1924).

La moneda y el cambio en España, Imprenta de los Hijos de J. A. García, Madrid, 1905. En esta obra se tratan las causas del quebranto del cambio en España. En opinión del autor la causa del mal de la moneda depreciada no ha sido el déficit de la balanza económica, sino que se ha producido por el abuso del papel moneda o por la alteración del valor relativo entre el oro y la plata. Sanz y Escartín atribuye el origen de la depreciación monetaria en España a que, cuando en 1872 comienza la depreciación de la plata, el Gobierno no hizo caso de las recomendaciones de la Junta Consultiva de Moneda y siguió inundando de plata la circulación debido, probablemente y según el autor, al beneficio que el Tesoro obtenía con tales acuñaciones de plata. Esa política monetaria condujo fatalmente a la desaparición del oro en España, obligando al Banco de España a la conversión de sus billetes únicamente en plata. Lo que llevó a que, entre 1896 y 1903, el cambio se elevara (depreciara) entre el $26 \%$ y el $35 \%$, de modo que los precios de todos los artículos subieron y la economía española entró de nuevo en una crisis importante, en 1905.

TUTAU y VERGES, Juan (1829-1893). Ministro de Hacienda en 1873 y 1874.

Las crisis monetarias, bursátiles, mercantiles e industriales, Editor Evaristo Ullastres, Barcelona (1886). Reconoce en este libro el autor que, a mediados del XIX, estábamos 
abocados a una gran perturbación monetaria ocasionada por la depreciación de la plata, que él atribuye a la preferencia por el oro, a la exagerada producción de plata, a su desmonetización en Alemania y a la suspensión en muchos países de las acuñaciones en plata a partir de 1873. Admite que la proscripción de la moneda de plata como instrumento de pago internacional ha provocado en España una elevación de los cambios (depreciación de la moneda) y el consiguiente aumento de los precios interiores. Ante la crisis monetaria en España no duda en admitir que habría que optar por acudir a la sustitución de la plata por el oro y propone en el libro los medios para afrontar dicha crisis.

\section{VÁZQUEZ QUEIPO DE LLANO, Vicente} (1804-1893). Senador vitalicio (1851). Miembro de la Junta Consultiva de Moneda. Miembro de la Real Academia de la Historia y Académico numerario de la Real Academia de Ciencias Exactas, Físicas y Naturales (1847). Contribuyó notablemente con su Ensayo sobre el sistema métrico y monetario (1859), sobre el que trabajó desde 1835 y con previa publicación del Proyecto de Ley para el caso español en 1847, así como con sus Tablas de logaritmos vulgares (1855).

La Cuestión del Oro reducida a sus justos límites y medios de sentar el sistema monetario sobre una sólida e inalterable base, Imprenta Nacional, Madrid, 1861. Vázquez Queipo trata aquí los efectos que la abundancia de oro podría provocar en su precio. Sobre este particular sostiene "[...]que a pesar de esta inmensa masa de oro[...] el oro no decaerá en su estimación[...]" (pp. 11 y 12), lo que le lleva a rechazar el argumento de que el aumento de precios experimentado a mediados del XIX se deba a una disminución del valor del oro. Y en cuanto a la inestabilidad del valor relativo entre el oro y la plata reconoce que es imposible establecer un valor relativo fijo, lo que le lleva a proponer la adopción en España de un patrón único sobre la base de la plata.

La crisis monetaria española considerada en sus causas, sus efectos y su remedio, Imprenta de José Cruzado, Madrid. 1866. En esta obra trata las causas de la crisis monetaria en España y, en contra de otras opiniones que afirman que la crisis monetaria española está provocada por la escasez de numerario metálico, que se produce como consecuencia de los pagos al exterior, Vázquez Quepo opina que la causa de dicha escasez de metálico se debe al incremento considerable de su demanda, producido por el descredito que sufre el papel fiduciario en circulación que es consecuencia de su inconsiderada e ilimitada emisión.

La cuádruple alianza monetaria considerada en su origen, objetos, ventajas e inconvenientes, e imposibilidad actual de su adopción en España, Imprenta La Reforma, Madrid. 1867. Vázquez Queipo pone en duda aquí la posibilidad de implantar la unidad monetaria internacional propuesta en la Convención celebrada en París en 1865, entre Francia, Italia, Suiza y Bélgica (Unión Monetaria Latina), porque considera que el valor de la moneda varía en los distintos puntos de la tierra. Además, aprovecha para manifestar que dicha Convención no innovó lo más mínimo los sistemas monetarios de esas cuatro naciones.

VIVER, Eudaldo (1854-1900). Dejó la carrera eclesiástica para dedicarse a la enseñanza del francés. Traductor de Shakespeare al español (1872). Finalmente se dedicó al estudio de las ciencias económicas.

Introducción al estudio de la cuestión monetaria, Tipografía de Luis Tarso, Barcelona, 1892. Viver comienza tratando en este libro los conceptos de utilidad, valor, precio y el dinero, así como las características de la moneda metálica. Sin embargo, los temas más interesantes se recogen en los capítulos VI y VIII. En el capítulo VI plantea las diferencias entre la aplicación del sistema monometálico y el bimetálico. Sobre tal cuestión opina Viver que la implantación de un doble patrón monetario exige mantener una relación fija entre el oro y la plata, lo cual, sostiene, ha fracasado siempre y añade que el doble patrón no es simultáneo sino alternativo. En el capítulo VIII trata sobre los problemas derivados de la inestabilidad del valor relativo entre el oro y la plata provocada por el notable aumento de la producción de ambos metales. Examina, asimismo, los acuerdos y propuestas de la Conferencia de París de 1865, que considera muy importante por las cuestiones tratadas y las doctrinas que allí se verificaron. También investiga las causas de la depreciación de la plata, que atribuye fundamentalmente a la decisión de Alemania de adoptar el patrón único oro, lo que llevó a la proscripción de la plata como instrumento de pago internacional, provocando graves efectos tanto sobre las transacciones españolas con el exterior como, especialmente, sobre el nivel de nuestros cambios. Y el capítulo finaliza explicando las medidas adoptadas 
por EE.UU. para defenderse de los problemas que le planteaba la depreciación de la plata en Europa.

Igualmente son de interés los capítulos IX y X que están dedicados a la implantación y desarrollo del monometalismo en Inglaterra y del bimetalismo en Francia. En el capitulo XII se estudian las causas de las crisis monetarias y económicas, estableciendo Viver que las crisis monetarias no son naturales sino artificiales y están provocadas por quienes monopolizan el mercado monetario, como es el caso de los bancos de emisión, los cuales, según sujeten o suelten las válvulas que regulan sus disponibilidades, pueden dar lugar a perturbaciones que unas veces se manifiestan en plétoras de dinero y otras, por el contrario, produciendo el vacío en la circulación monetaria.

Mención particular de la situación monetaria española realiza Viver en el capítulo V, donde establece las circunstancias que llevaron a las reformas de 1848, 1864, 1868 y 1876 , así como todo lo relativo a la reforma de 1868 . $\mathrm{Y}$ en el capítulo XIV critica el exceso de las emisiones de billetes en España como recurso para financiar y cubrir sus presupuestos, en lugar de recurrir al crédito público, lo que le lleva a considerar la necesidad de una nueva política fiscal. Además, analiza también las consecuencias que se producen en España por carecer de oro para atender los pagos con el exterior. 